- Published experiments with small quantum devices are starting to approach this faultiness threshold, notes Mosca. And because secretive organizations such as the NSA are keenly interested in the technology, it is widely assumed that these published results do not represent the cutting edge of research. "We have to assume there's going to be people that are a few years ahead of what's available in the public literature," says Mosca. "You can't wait for the headlines in The New York Times to have your plan in place."

The safety of today's Internet traffic relies in part on a type of encryption called publickey cryptography - which includes RSA - to establish secret communication between users. A sender uses a freely available digital key to lock a message, which can be unlocked only with a secret key held by the recipient. The security of RSA depends on the difficulty of breaking up a large number into its prime factors, which serve as its secret key. In general, the larger the number, the harder this problem is to solve.

Researchers believe that it takes existing computers a long time to factorize big numbers, partly because no one has yet discovered how to do it quickly. But quantum computers could factorize a large number exponentially faster than any conventional computer, and this nullifies RSA's reliance on factoring being difficult.

Several options already exist for new publickey cryptosystems. These replace the factoring problem with other difficult mathematics problems that are not expected to yield to quantum computers. Although these systems are not perfectly safe, researchers think that they are secure enough to protect secrets from quantum computers for all practical purposes.

One such system is lattice-based cryptography, in which the public key is a grid-like collection of points in a high-dimensional mathematical space. One way to send a secret message is to hide it some distance from a point in the lattice. Working out how far the encrypted message is to a lattice point is a difficult problem for any computer, conventional or quantum. But the secret key provides a simple way to determine how close the encrypted message is to a lattice point.

A second option, known as McEliece encryption, hides a message by first representing it as the solution to a simple linear algebra problem. The public key transforms the simple problem into one that seems much more difficult. But only someone who knows how to undo this transformation - that is, who has the private key - can read the secret message.

One drawback of these replacements is that they require up to 1,000 times more memory to store public keys than existing methods, although some lattice-based systems have keys not much bigger than those used by RSA. But both methods encrypt and decrypt data faster than today's systems, because they rely on simple multiplication and addition, whereas RSA uses more-complex arithmetic.

PQCRYPTO, a European consortium of quantum-cryptography researchers in academia and industry, released a preliminary report on 7 September recommending cryptographic techniques that are resistant to quantum computers (see go.nature.com/5kellc). It favoured the McEliece system, which has resisted attacks since 1978, for public-key cryptography. Tanja Lange, head of the €3.9-million (US\$4.3-million) project, favours the safest possible choices for early adopters. "Sizes and speed will improve during the project," she says, "but anybody switching over now will get the best security." -

\title{
Germany claims success for elite universities drive
}

\section{Report praises $€ 4$.6-billion scheme to make leading universities more competitive - but some smaller institutions have done just as well.}

\section{BY QUIRIN SCHIERMEIER \& RICHARD VAN NOORDEN}

$\mathrm{F}$ or a decade, Germany's government has been trying to explode the myth that all the country's universities are equal. In 2006, it launched an 11-year, €4.6-billion (US\$5-billion) programme that aimed to make the best German universities more competitive with the likes of Oxford, Cambridge and Harvard. The campaign, called the Excellence Initiative, led to 14 institutions gaining the unofficial label of 'elite'.

A 3 September report by Germany's main research-funding agency, the DFG - which administers the initiative together with Germany's science council - suggests that the cash influx is paying off. Still, a German equivalent of the US Ivy League may be slow to form. An analysis by Nature's news team shows that some universities less favoured by the initiative have improved just as quickly as the elites when it comes to generating highly cited work. "It doesn't require the 'elite' label to produce good research in Germany," says Alfred Forchel, president of the University of Würzburg, an institution that has kept pace without top-up funds.

The DFG sees this as positive. "The Excellence Initiative has met expectations," says Dorothee Dzwonnek, DFG secretary-general. "And it has not weakened universities which don't directly benefit from it." But some critics say that the scheme has benefited administrators more than scientists. And a huge increase in research funding across Germany over the past decade makes it difficult to tease out the influence of the initiative on the country's improvement.

The DFG report, an analysis of funding in German universities that is released every three years, marks the first attempt to measure preliminary outcomes of the initiative. In 2011-13 alone, 45 universities received a total of more than $€ 1$ billion for running international graduate schools and setting up specific clusters of excellence. A subset also each received an extra $€ 10$ million to $€ 14$ million a year for 'institutional strategies' to strengthen the university as a whole - the most prestigious part of the competition (see Nature 487, 519-521; 2012).

The elite group includes some of Germany's largest and best-equipped research universities, such as the Ludwig Maximilian University of Munich and RWTH Aachen University. The report shows that the elites dominate when it comes to winning competitive grants from the DFG. As a group, they secured more than $40 \%$ of the agency's total funding from 2011-13. However, the same 14 institutions won almost the same share of DFG funding in 2002-04, before the initiative had launched.

Scientific output is booming at the 45 universities that got cash out of the Excellence Initiative, the DFG report points out. They have boosted their output by $43 \%$ in chemistry and physics since 2002 , more than the $34 \%$ increase in these subjects by all German universities. 


\section{GERMANY RISING}

Germany's research articles are rapidly becoming more highly cited. But some of the country's smaller universities are matching the rise of its 'elite' institutions.

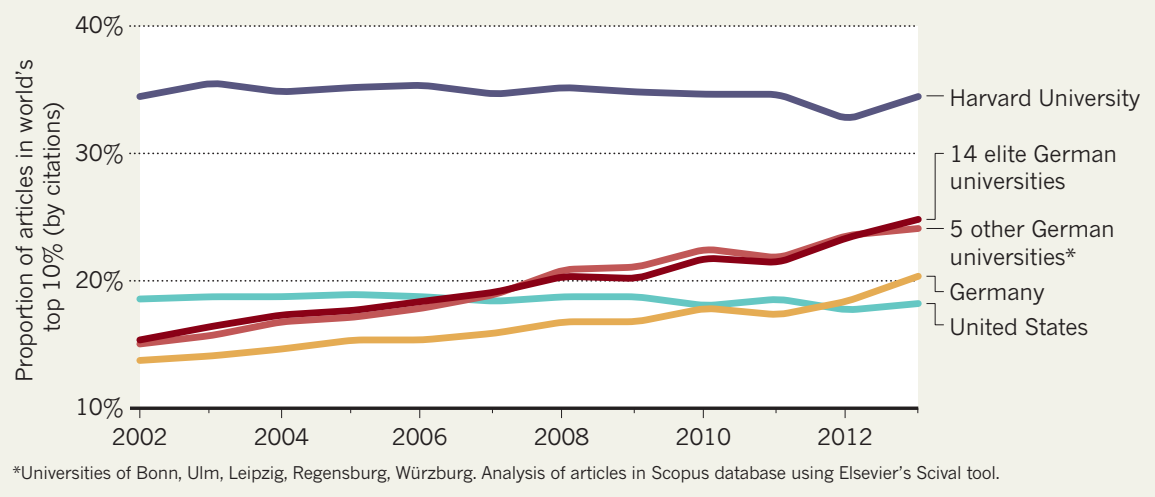

And a further analysis by Nature finds that the 14 elites alone now produce $35 \%$ of Germany's total articles, up from a share of $29 \%$ in 2002.

\section{THE REST CHASE THE BEST}

But the Excellence Initiative may not be separating the elites from the rest when it comes to the quality of research papers. Nature's analysis shows that almost one-quarter of articles from the elites are now in the world's top $10 \%$ by citations - up from one-sixth 12 years ago. Yet it also shows that some other German universities that received much less funding, or no top-up funds, have matched this rise (see 'Germany rising'). That is enlightening, says Karl Ebeling, president of the University of Ulm, which had little success in the initiative but is higher in some international rankings than elite universities in Bremen and Konstanz, for instance.

Björn Brembs, a neurobiologist at the University of Regensburg, thinks that the unclear impact of the initiative on creating elites is because the cash was poorly spent. He has delved into German employment statistics and found evidence, he says, of booming academic bureaucracy. "For every scientist who has been recruited thanks to the Excellence Initiative, four new administrative positions were created," he says. "It is hardly surprising that elite institutions have no research advantage over some other universities when the group that benefits most from the Excellence Initiative is not involved in science."

The DFG says that it has not looked at how the programme may have burdened university administrations. "It has attracted 4,000 talented foreign scientists to German universities and it has greatly increased these universities' scholarly output," says Dzwonnek. "From our point of view, this is a real success." Many agree that the competition, despite ambiguity over its measurable impacts, has served German science well. It was a positive shock to Germany's structurally conservative science system, says Jakob Edler, executive director of the Manchester Institute of Innovation Research, UK.

The results of a comprehensive evaluation of the Excellence Initiative by an international panel of experts are due in January 2016. The federal government and Germany's 16 states, which have tentatively agreed to continue the programme, will then decide about its future. "The Excellence Initiative promotes fresh ideas and new collaborations. I do hope it continues beyond 2017," Forchel says. 